\title{
Inactivity during COVID-19 Quarantine and its Effects in Strength and Functional Parameters in Elderly: A Case-Study
}

Júlio Benvenutti Bueno de Camargo ${ }^{1 *}$, Rubem Machado Filho ${ }^{1}$, Felipe Alves Brigatto ${ }^{1}$, Danilo Rodrigues Batista ${ }^{1}$, Paulo Henrique Barbosa ${ }^{1}$, Guilherme Borsetti Businari', Jhenipher Moniky Rosolem $^{1}$, Rafael Sakai Zaroni ${ }^{1}$, Evandro Murer ${ }^{2}$, Gustavo Zorzi Fioravante ${ }^{1}$, Daniel Alves Corrêa ${ }^{1}$, Diego Pereira Jeronimo ${ }^{3}$, Anderson Martelli", Moisés Diego Germano ${ }^{1}$, Wellington Gonçalves Dias ${ }^{1}$, Victor Augusto Ramos Fernandes ${ }^{5}$, Luan Oenning $\mathrm{Co}^{6}$ and Tiago Volpi Braz ${ }^{1}$

${ }^{1}$ Methodist University of Piracicaba - UNIMEP, Brazil

${ }^{2}$ Faculty of Americana - FAM, Brazil

${ }^{3}$ State University of Campinas - UNICAMP, Brazil

${ }^{4}$ UniMogi - UNIMOGI, Brazil

${ }^{5}$ Faculty of Medicine of Jundiai - FMJ, Brazil

${ }^{6}$ Adventist University Sao Paulo - UNASP, Brazil

*Corresponding author: Júlio Benvenutti Bueno de Camargo, Methodist University of Piracicaba (UNIMEP), College of Health Science (FACIS), Graduate Program in Science of Human Movement, Campus Taquaral, Rodovia do Acucar, $\mathrm{km}$ 156, Piracicaba (SP), CEP: 13400-911, Brazil

\begin{abstract}
Background: Coronavirus 2019 (COVID-19) may be defined as a disease with pandemic characteristics. Although social distance may be able to combat the spreading of the virus, negative repercussions in general population's health may occur, especially in older people. Then, the aim of the present case-study was to assess the effects of a 6 month-detraining period in strength and functional parameters of a previously strength-trained elderly woman.

Methods: Lower limbs maximal strength (one maximum repetition test [1RM]), timed-up and go (TUG) and Sit to Stand (STS) tests were performed pre and post the inactivity period.

Results: Relevant reductions in 1RM (-25.9\%) and in the number of repetitions performed in STS $(-28.5 \%)$ were observed. In addition, the performance in TUG (+30.5\%) was also impaired after the detraining phase.

Conclusion: In conclusion, physical inactivity during the COVID-19 quarantine induced important reductions in strength and functional parameters in the elderly participant. Then, it can be recommendable that older people initiate or maintain their RT practices even at home during this period in order to maintain optimal general health and quality of life.
\end{abstract}

\section{Introduction}

Coronavirus 2019 (COVID-19) may be defined as a disease with pandemic characteristics. During 2020year, COVID-19 has become a public health emergency of worldwide proportions [1], requiring then strong actions by health organizations and professionals. However, due to the absence of an effective therapeutic intervention and the significant transmission rate extensively reported, the major form of combating and reducing the number of infections is by adopting social distance practices, which might also help to ensure that health systems would be able to properly meet the high health care-related demands during this period. Although such practice has shown to better control the virus' spreading [1], it may also induce significant negative repercussions in worldwide population, special related to the increasing physical inactivity level.

Compared to young individuals, older adults usually present lower levels of physical activity [2]. Indeed, the ability to perform distinct daily living and work physical activities, is considerably decreased during human

Citation: Camargo JBB, Filho RM, Brigatto FA, Batista DR, Barbosa PH, et al. (2020) Inactivity during COVID-19 Quarantine and its Effects in Strength and Functional Parameters in Elderly: A Case-Study. Int J Sports Exerc Med 6:180. doi.org/10.23937/2469-5718/1510180

Accepted: December 29, 2020; Published: December 31, 2020

Copyright: (C) 2020 Camargo JBB, et al. This is an open-access article distributed under the terms of the Creative Commons Attribution License, which permits unrestricted use, distribution, and reproduction in any medium, provided the original author and source are credited. 


\section{Detraining period}

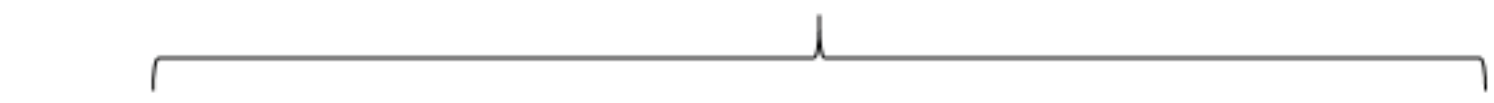

$\operatorname{March} / 20$

September $/ 20$

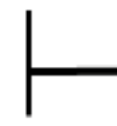

AM
1RM
TUG
STS

AM

1RM

TUG

Figure 1: Experimental design of the detraining period during COVID-19 quarantine.

AM: Anthropometric measurements; 1RM: One repetition maximum test in the squat exercise; TUG: Timed up and go test; STS: Sit to stand test.

aging as a consequence of several neuromuscular impairments. Reduced levels of functional capacity in older adults are related to lower quality of life [3], and an increasing risk of falling [4]. In addition, such population also experiences significant reductions in muscle strength, which reflects in an impaired performance of daily activities (e.g. stand up; walking; climbing stairs). Then, it is plausible to assume that older individuals home-isolated during quarantine of COVID-19 may present even more reduced levels of physical activity [5]. Additionally, older individuals normally engaged in Resistance Training (RT) programs present larger reductions in muscle strength and size when exposed to a detraining period compared to their young counterparts and also demand higher amounts of training volume in order to retain RT-induced previous adaptations [6].

Them, the aim of the present case-study was to assess the detraining effects of social isolation during COVID-19 and the consequent cessation of RT in functional and strength parameters in an elderly woman. The initial hypothesis was that the inactivity period would induce high-magnitude decreases in the variables assessed.

\section{Methods}

\section{Case description}

The participant in this case was a 66-year woman (Body mass: $81.8 \mathrm{~kg}$; Height: $168.8 \mathrm{~cm}$; Body mass index: 29.0) who was engaged in RT practice for the last 5 years. Previous to the isolation period, RT program was performed $3 x /$ week, with training loads ranging from 8 to 12 maximum repetitions (RM) for each set. In each training session, 8 exercises (Smith Machine Back Squat; Seated row; Machine Leg Press; Machine bench press; Knee extension; Knee flexion; Cable elbow extension; Shoulder abduction) were performed with 2-minutes rest intervals afforded between sets and exercises. No chronic disease was reported before the beginning of the study. Also, the participant was free from any muscle or skeletal injury and negatively responded all the questions in the PAR-Q. Before initiating the study, the participant was informed about all the details and procedures adopted during the study, read and signed a consent form. In addition, the voluntary was also familiarized with the testing procedures. The study followed all the norms of the Brazilian National Health Council (CNS), according to the 510/2016 resolution.

\section{Procedures}

The detraining period lasted from March to September of the 2020 year. During this period, participant abstained for every strength training practice, with a self-reporting reduction in physical activity levels. Assessments of anthropometric parameters, muscle strength and functional fitness were performed for both pre and post the social isolation period (Figure 1). A 10-minute period was afforded between each test. The assessments in the pre-moment were performed 72 hours after the last RT session. All testing procedures were supervised by the same researcher. The participant was provided with a standardized verbal encouragement during the tests.

\section{Anthropometric parameters}

Height (centimeters) and body mass (kilograms) were assessed before and after the detraining period. To evaluate height and body mass, a stadiometer/mechanical scale (SECA, model 220, Germany) with precisions of $0.1 \mathrm{~cm}$ and $0.1 \mathrm{~kg}$, respectively, was used. Both parameters were assessed prior to all physical performance tests. The Body Mass Index (BMI) was also calculated $\left(\mathrm{kg} / \mathrm{m}^{2}\right)$.

\section{Maximal dynamic strength}

One repetition maximum test (1RM) in Smith Machine Back Squat $\left(1 \mathrm{RM}_{\text {SQUAT }}\right)$ exercise was adopted in order to assess lower limbs maximal strength. The par- 
Table 1: Pre and post 6-month-detraining period values for anthropometric measurements.

\begin{tabular}{|l|l|l|l|}
\hline Variables & pre & post & $\Delta(\mathbf{\%})$ \\
\hline BM $(\mathrm{kg})$ & 81.8 & 83.6 & 2.2 \\
\hline Height $(\mathrm{cm})$ & 168.8 & 168.9 & 0.05 \\
\hline BMI & 29 & 29.6 & 2 \\
\hline WC $(\mathrm{cm})$ & 81.5 & 84.2 & 3.31 \\
\hline
\end{tabular}

BM: Total body mass; BMI: Body Mass Index; WC: Waist Circumference; kg: kilograms; cm: centimeters

Table 2: Pre and post 6-month-detraining period values for the variables assessed.

\begin{tabular}{|l|l|l|l|}
\hline Variables & pre & post & $\Delta$ (\%) \\
\hline 1RM $(\mathrm{kg})$ & 54.0 & 40.0 & -25.9 \\
\hline TUG $(\mathrm{s})$ & 7.2 & 9.4 & 30.5 \\
\hline STS $($ reps) & 14 & 10 & -28.5 \\
\hline
\end{tabular}

1RM: One repetition maximum test in the back squat exercise; TUG: Time-up and go test; STS: Sit-to-stand test; kg: kilograms; s: seconds; reps: number of repetitions

ticipant was already previously familiarized with the exercise and the testing procedures during the years of training. All procedures were performed according to previous recommendations [7]. The data are expressed in kilograms ( $\mathrm{kg})$.

\section{Timed Up and Go test (TUG)}

The test consisted of standing up from a chair, walking $2.44 \mathrm{~m}$, and turning and returning to the initial seated position. The test was administered according to previously described instructions from Pereira, et al. [8]. The data are expressed in seconds (s).

\section{Sit to Stand test (STS)}

The test consisted of standing up from a chair and returning to the initial seated position, completing as many repetitions as possible in $30 \mathrm{~s}$. The test was administered according to the recommendations of Hallage, et al. [9].

\section{Results}

The pre and post-inactivity anthropometric measures are presented in Table 1. For body mass and waist circumference, absolute increases of $1.8 \mathrm{~kg}$ and $2.7 \mathrm{~cm}$ were observed, respectively.

The pre and post-inactivity strength and functional measures are presented in Table 2. For $1 \mathrm{RM}_{\text {SQUAT }}$, an absolute decrease of $14.0 \mathrm{~kg}$ was observed. For TUG, an increase of $2.2 \mathrm{~s}$ was observed and, for STS, an absolute decrease of 4 repetitions performed was observed from the pre to post-inactivity period.

\section{Discussion}

The aim of the present case-study was to assess the effects of inactivity during the COVID-19 quarantine period in strength and functional parameters of an el- derly woman. Confirming the initial hypothesis, large reductions were observed in the dependent variables assessed.

Studies investigating the effects of detraining in elderly population during this pandemic period may bring relevant information about the eventual deleterious effects of the inactivity in general health-related parameters and help health-professionals to better implement exercise-programs during this phase in order to counteract these undesired negative outcomes. However, due to the scarce number of similar investigations, possible comparisons with our results become limited.

Elevated muscle strength-levels are strongly correlated to lower risk of all cause-mortality $[10,11]$. Elderly people can beneficiate from RT-programs in order to improve their performance in daily tasks, as walking, standing up, climbing stairs [11], which in turn may also increase independence-levels. In addition, higher strength levels also seem to negatively associate with depressive symptoms [12] and risk of falls [13]. Then, the cessation of RT-programs may bring relevant negative effects in the quality of life of these population.

The effects of muscle strength-detraining seem to be more pronounced in the older population compared to their younger counterparts. Decreases of higher magnitude have been described in muscle cross sectional area, where older individuals seem to demand a higher training volume (number of sets) in order to maintain the morphological adaptations previously acquired during a RT program when compared to young adults [6]. However, muscle strength seems to decrease in a smaller rate when compared to muscle size. Indeed, a 32-week detraining phase was not sufficient to reduce maximal strength to baseline levels in older subjects [6]. The maintenance of strength gains during a detraining period has been mainly attributed to neural adaptations, as muscle size returns more rapidly to pre-training levels during detraining [14].

Comparisons between the decreases in maximal strength observed in our study and other investigations must be done with some caution, especially due to the long detraining period adopted and the training experience of the participant of this study. Pereira, et al. [8] and Blocquiaux, et al. [15], for example, reported a reduction of $18.1 \%$ and $10.0 \%$ (respectively) in maximal strength of lower limbs in elderly individuals. The short detraining period ( 6 weeks and 12 weeks, respectively) and the participants' lack of previous experience in RT may help to explain the distinct results between the current study and the aforementioned investigations.

It is also interesting to note that the relative decreases induced by the detraining period were higher in strength compared to functional parameters in the current study. These results corroborate previous findings from Pereira, et al. [8]. However, some methodological 
differences must be considered in an attempt to compare both studies, since high-speed power training and a short detraining period (only 6 weeks) was adopted during the intervention of Pereira, et al. [8]. Then, based on the smaller percentage decrements in functional task (STS) observed in the latter $(-2.8 \%)$ compared to the current study $(-28.5 \%)$, it can be suggested that older individuals can experience smaller decrements in functional performance during the cessation of the training program when submitted to high-speed RT-protocols, although further investigations should be implemented in order to confirm such hypothesis.

\section{Conclusions}

Based on the results observed in the present study, the inactivity period during the COVID-19 period may bring relevant decrements in strength and functional parameters for the elderly individual, which, in turn, may induce a higher level of dependence and an increased risk of falls in this population. Then, health professionals should be aware of these effects and aim to implement strategies to minimize them.

It is important to note that this is only a single case study and its significance should be judged on that basis. Then, future studies with large samples and proper statistical analysis must help to clarify these findings. However, this case study raises a question: what would be the exact damage caused by social isolation during the COVID-19 period in elderly's health? This question opens the door for further discussion and investigation.

Considering the significant positive effects usually induced by physical activity programs in elderly individuals' health, it is recommendable that this population should aim to initiate or maintain their RT practices even at home. Using adaptable weights, elastic bands, and on-line supervised programs might help to reduce the harmful effects of physical inactivity during social distance of COVID-19.

\section{References}

1. Shereen MA, Khan S, Kazmi A, Bashir N, Siddique R (2020) COVID-19 infection: Origin, transmission, and characteristics of human coronaviruses. J Adv Res 24: 91-98.

2. Meijer EP, Goris AH, Wouters L, Westerterp KR (2001) Physical inactivity as a determinant of the physical activity level in the elderly. Int J Obes Relat Metab Disord 25: 935-939.
3. Oztürk I, Toker S, Ertürer E, Aksoy B, Seçkin F (2008) Analysis of risk factors affecting mortality in elderly patients (aged over 65 years) operated on for hip fractures. Acta Orthop Traumatol Turc 42: 16-21.

4. Moreira NB, Rodacki ALF, Pereira G, Bento PCB (2018) Does functional capacity, fall risk awareness and physical activity level predict falls in older adults in different age groups? Arch Gerontol Geriatr 77: 57-63.

5. Belavý DL, Gast U, Daumer M, Fomina E, Rawer R, et al. (2013) Progressive adaptation in physical activity and neuromuscular performance during $520 \mathrm{~d}$ confinement. PLoS One 8: 60090.

6. Bickel CS, Cross JM, Bamman MM (2011) Exercise dosing to retain resistance training adaptations in young and older adults. Med Sci Sports Exerc 43: 1177-1187.

7. Baechle TR, Earle RW (III) (2008) Essentials of strength training and conditioning. Human Kinetics, Champaign.

8. Pereira A, Izquierdo M, Silva AJ, Costa AM, González-Badillo JJ, et al. (2012) Muscle performance and functional capacity retention in older women after high-speed power training cessation. Exp Gerontol 47: 620-624.

9. Hallage T, Krause MP, Haile L, Miculis CP, Nagle EF, et al. (2010) The effects of 12 weeks of step aerobics training on functional fitness of elderly women. J Strength Cond Res 24: 2261-2266.

10. Ling CH, Taekema D, de Craen AJ, Gussekloo J, Westendorp RG, et al. (2010) Handgrip strength and mortality in the oldest old population: the Leiden 85-plus study. CMAJ 182: 429-435.

11. Haraldstad K, Rohde G, Stea TH, Lohne-Seiler H, Hetlelid K, et al. (2017) Changes in health-related quality of life in elderly men after 12 weeks of strength training. Eur Rev Aging Phys Act 14: 8.

12. Marques A, Gomez-Baya D, Peralta M, Frasquilho D, Santos T, et al. (2020) The Effect of Muscular Strength on Depression Symptoms in Adults: A Systematic Review and Meta-Analysis. Int J Environ Res Public Health 17: 5674 .

13. Pizzigalli L, Filippini A, Ahmaidi $S$, Jullien $H$, Rainoldi $A$ (2011) Prevention of falling risk in elderly people: the relevance of muscular strength and symmetry of lower limbs in postural stability. J Strength Cond Res 25: 567-574.

14. Häkkinen K, Alen M, Kallinen M, Newton RU, Kraemer WJ (2000) Neuromuscular adaptation during prolonged strength training, detraining and re-strength-training in middle-aged and elderly people. Eur J Appl Physiol 83: 51-62.

15. Blocquiaux S, Gorski T, Van Roie E, Ramaekers M, Van Thienen R, et al. (2020) The effect of resistance training, detraining and retraining on muscle strength and power, myofibre size, satellite cells and myonuclei in older men. Exp Gerontol 133: 110860. 